\title{
Kink topology control by high-frequency external forces in nonlinear Klein-Gordon models
}

\author{
R. Alvarez-Nodarse,,$^{1,2, *}$ N. R. Quintero, ${ }^{1,3, \dagger}$ and F. G. Mertens ${ }^{4, \ddagger}$ \\ ${ }^{1}$ Instituto de Matemáticas de la Universidad de Sevilla (IMUS), Universidad de Sevilla, 41012 Sevilla, Spain \\ ${ }^{2}$ Departamento de Análisis Matemático, Universidad de Sevilla, apdo. 1160, E-41080, Sevilla, Spain \\ ${ }^{3}$ Departamento de Física Aplicada I, E.P.S., Universidad de Sevilla, Virgen de África 7, 41011, Sevilla, Spain \\ ${ }^{4}$ Physikalisches Institut, Universität Bayreuth, 95440 Bayreuth, Germany
}

(Received 3 August 2014; published 20 October 2014)

\begin{abstract}
A method of averaging is applied to study the dynamics of a kink in the damped double sine-Gordon equation driven by both external (nonparametric) and parametric periodic forces at high frequencies. This theoretical approach leads to the study of a double sine-Gordon equation with an effective potential and an effective additive force. Direct numerical simulations show how the appearance of two connected $\pi$ kinks and of an individual $\pi$ kink can be controlled via the frequency. An anomalous negative mobility phenomenon is also predicted by theory and confirmed by simulations of the original equation.
\end{abstract}

DOI: 10.1103/PhysRevE.90.042916

PACS number(s): 05.45.Yv, 05.60.Cd

\section{INTRODUCTION}

It is well known that the sine-Gordon equation is an integrable system [1]. It possesses infinitely many soliton solutions as well as infinitely many conserved quantities. Moreover, the soliton interactions are elastic. These properties have been used, in the case of the sine-Gordon system, to describe the nonlinear dynamics of biopolymers [2]. Double sine-Gordon and $\phi^{4}$ equations are nearly integrable, and only a few solutions are known [3]. These solutions are called solitary waves instead of solitons because these waves interact inelastically [4-8].

From the physical point of view, it is important to include the dissipation and external forces in these mathematical models. These perturbations should be small to prevent the destruction of the solitary waves [9]. These perturbed equations currently model the propagation of fluxons in long Josephson junctions [10,11], dislocations in solids, ferromagnetic systems, etc. (see references in the review paper [12]). In particular, the double sine-Gordon equation has been used as a model for low-dimensional magnetic systems (see Ref. [13] and references therein) and to describe the defect propagation in hydrogen-bonded molecular chains $[14,15]$.

Among all possible perturbations, the external (nonparametric) and parametric periodic forces have been widely used [16-24]. In particular, when the solitary waves are strongly driven by high-frequency forces, it has been shown that there is a range of parameters for which the kink cannot exist [16] in the damped parametrically driven $\phi^{4}$ system. Phenomena of a more interesting nature appear in the damped parametric and additive driven sine-Gordon equation. Indeed, in the former case, a $2 \pi$ kink, a solution consisting of two connected $\pi$ kinks, was predicted by the theory and confirmed by simulations [20]. In the latter case, a $\pi$ kink was found [23].

In the current work, the damped double sine-Gordon equation, driven by both external (nonparametric) and parametric forces is studied. It should be noted that, in the

*ran@us.es

†niurka@us.es

${ }^{\ddagger}$ Franz.Mertens@uni-bayreuth.de high-frequency limit, we show that the appearance of one of the two connected $\pi$ kinks can be controlled via the frequency. A similar phenomenon has been reported in recent experiments on helical ribbons formed from a paramagnetic colloid in a dynamic magnetic field, where the particular form of the domain walls are controlled via the frequency and the amplitudes of the forces [25].

In order to achieve our goal, we consider the following perturbed double sine-Gordon equation:

$$
\begin{aligned}
& \frac{\partial^{2} \phi}{\partial t^{2}}-\frac{\partial^{2} \phi}{\partial x^{2}}+\mu \sin \phi+\lambda \sin 2 \phi \\
& \quad=f-\gamma \frac{\partial \phi}{\partial t}+\epsilon_{1} \sin \phi \cos \omega t-\epsilon_{2} \cos \phi \sin \omega t+\epsilon_{3} \sin \omega t,
\end{aligned}
$$

where all variables and parameters are in dimensionless form. In particular, $\mu$ and $\lambda$ are parameters of the double sine-Gordon equation, while $f$ is a constant force, $\epsilon_{j}(j=1,2,3)$ are the amplitudes of the $T=2 \pi / \omega$ periodic forces, and $\gamma$ is the dissipation coefficient.

In order to describe the dynamics of solitary waves of this equation, a method of averaging, introduced in Ref. [16], is applied. Although this method of averaging $[18,22,25]$ is less general than that developed in Ref. [20], it is shown here that this method leads, not only to very interesting theoretical results, but also to good agreement with the numerical simulations of Eq. (1). Moreover, we show that both methods yield the same results in the first-order correction.

The paper is organized as follows. In Sec. II, a method of averaging for a more general equation is developed, namely, the damped and driven nonlinear Klein-Gordon equation,

$$
\frac{\partial^{2} \phi}{\partial t^{2}}-\frac{\partial^{2} \phi}{\partial x^{2}}+V^{\prime}(\phi)=g(t, \phi)-\gamma \frac{\partial \phi}{\partial t},
$$

with potential $V(\phi)$, driven by constant, external periodic (nonparametric), and parametric forces

$$
g(t, \phi)=f+\epsilon_{1} A(\phi) \cos \omega t+\epsilon_{2} B(\phi) \sin \omega t+\epsilon_{3} \sin \omega t
$$

for sufficiently large values of $\omega$. Interestingly, in the highfrequency limit, the perturbation can be large, i.e., $\epsilon_{1} \gg 1$, 
$\epsilon_{2} \gg 1$, and $\epsilon_{3} \gg 1$. In Sec. III, for a particular choice of the functions $A(\phi)$ and $B(\phi)$, the kink dynamics of Eq. (1) is analyzed by means of an effective potential. In Sec. IV the simulations of Eq. (1) are compared with the simulations of the corresponding effective equation obtained within the framework of a method of averaging. In Sec. V the phenomenon of absolute negative mobility of the kinks is explored. Finally, in Sec. VI the main conclusions of the work are summarized. A numerical analysis of the spectrum of linear excitations around the static kink solution in the double-sine Gordon equation is provided in the Appendix.

\section{THE METHOD OF AVERAGING FOR THE DAMPED NONLINEAR KLEIN-GORDON EQUATION}

In order to study the dynamics of kink solutions of Eqs. (2) and (3), we use the so-called method of averaging [16,18,26]. The main idea of this method is to separate fast and slow parts of the solution of Eq. (2). As a result, an effective partial differential equation is obtained, where the periodic forces do not appear explicitly but affect the effective potential. We apply the approach developed in Ref. [16], where the wellknown method of averaging for a pendulum motion under a high-frequency parametric force [26] was generalized to the $\phi^{4}$ chain with loss. This method is then applied to the sine-Gordon equation with the aim of modeling the Josephson junctions [19].

Equations (2) and (3) can be rewritten as

$$
\begin{aligned}
& \frac{\partial^{2} \phi}{\partial t^{2}}-\frac{\partial^{2} \phi}{\partial x^{2}}+V^{\prime}(\phi) \\
& \quad=f-\gamma \frac{\partial \phi}{\partial t}+\epsilon_{1} A(\phi) \cos \omega t+\left[\epsilon_{2} B(\phi)+\epsilon_{3}\right] \sin \omega t
\end{aligned}
$$

where, from henceforth, $\omega$ is considered sufficiently large (the meaning of large frequency is clarified later in the paper).

The first step of the method of averaging used here consists of splitting the solution of Eq. (4), $\phi(x, t)$, into two components: a slowly varying function $\Phi(x, t)$ and a fast and small varying function $\zeta(t)[16,18,26]$. The function $\zeta$ has zero mean and describes the small (and fast) oscillations around the slowly varying function $\Phi$ and is assumed to be independent of $x[16,18]$. This assumption is confirmed by simulations.

Substituting $\phi(x, t)=\Phi(x, t)+\zeta(t)$ into (4), we obtain

$$
\begin{aligned}
\frac{\partial^{2} \Phi}{\partial t^{2}}-\frac{\partial^{2} \Phi}{\partial x^{2}}+\frac{\partial^{2} \zeta}{\partial t^{2}}+\gamma \frac{\partial \Phi}{\partial t}+\gamma \frac{\partial \zeta}{\partial t}+V^{\prime}(\Phi+\zeta) \\
=f+\epsilon_{1} A(\Phi+\zeta) \cos \omega t+\epsilon_{2} B(\Phi+\zeta) \sin \omega t \\
\quad+\epsilon_{3} \sin \omega t
\end{aligned}
$$

In order to proceed, it is assumed that $\zeta$ is sufficiently small to obtain

$$
\begin{aligned}
& V^{\prime}(\Phi+\zeta)=V^{\prime}(\Phi)+V^{\prime \prime}(\Phi) \zeta+V^{\prime \prime \prime}(\Phi) \frac{\zeta^{2}}{2}+\cdots \\
& A(\Phi+\zeta)=A(\Phi)+A^{\prime}(\Phi) \zeta+\cdots \\
& B(\Phi+\zeta)=B(\Phi)+B^{\prime}(\Phi) \zeta+\cdots
\end{aligned}
$$

and then (5) becomes

$$
\begin{gathered}
\frac{\partial^{2} \Phi}{\partial t^{2}}-\frac{\partial^{2} \Phi}{\partial x^{2}}+\frac{\partial^{2} \zeta}{\partial t^{2}}+V^{\prime}(\Phi)+V^{\prime \prime}(\Phi) \zeta+V^{\prime \prime \prime}(\Phi) \frac{\zeta^{2}}{2}+\cdots \\
=f-\gamma \frac{\partial \Phi}{\partial t}-\gamma \frac{\partial \zeta}{\partial t}+\epsilon_{1}\left[A(\Phi)+A^{\prime}(\Phi) \zeta\right] \cos \omega t \\
\quad+\epsilon_{2}\left[B(\Phi)+B^{\prime}(\Phi) \zeta\right] \sin \omega t+\epsilon_{3} \sin \omega t+\cdots
\end{gathered}
$$

The next step consistently separates the equations of motion for $\zeta$ and $\Phi$. Following Refs. [16,26], we collect, on one hand, the fast varying terms and, on the other hand, the slowly varying terms. Assuming that $\epsilon_{j} \sim \omega, j=1,2,3$ and $\dot{\zeta} \propto \omega$, and that $\ddot{\zeta} \propto \omega^{2}$ since $\zeta$ oscillates with a frequency $\omega$, it can be deduced that the evolution of $\zeta$ is ruled by the Newtonian equation

$$
\begin{aligned}
\frac{\partial^{2} \zeta(t)}{\partial t^{2}}= & -\gamma \frac{\partial \zeta(t)}{\partial t}+\epsilon_{1} A(\Phi) \cos \omega t \\
& +\epsilon_{2} B(\Phi) \sin \omega t+\epsilon_{3} \sin \omega t
\end{aligned}
$$

where the periodic forces act directly on $\zeta$. Since $\Phi$ is a slowly varying function in comparison with $\zeta$, we can further assume that $\Phi$ is a constant, and hence Eq. (7) can be solved, which yields

$$
\begin{aligned}
\zeta(t)= & C_{1}+C_{2} e^{-\gamma t}+\frac{\epsilon_{1} A(\Phi) \gamma-\left[\epsilon_{2} B(\Phi)+\epsilon_{3}\right] \omega}{\omega\left(\gamma^{2}+\omega^{2}\right)} \sin (\omega t) \\
& -\frac{\epsilon_{1} A(\Phi) \omega+\left[\epsilon_{2} B(\Phi)+\epsilon_{3}\right] \gamma}{\omega\left(\gamma^{2}+\omega^{2}\right)} \cos (\omega t)
\end{aligned}
$$

where $C_{1}$ and $C_{2}$ are integration constants. After a transient time, $t \gg \tau=1 / \gamma$, then the second term, proportional to $C_{2}$, can be disregarded. Moreover, the assumption $\langle\zeta\rangle=0$ implies $C_{1}=0$. Therefore, Eq. (8) reduces to

$$
\begin{aligned}
\zeta(t)= & \frac{\epsilon_{1} A(\Phi) \gamma-\left[\epsilon_{2} B(\Phi)+\epsilon_{3}\right] \omega}{\omega\left(\gamma^{2}+\omega^{2}\right)} \sin (\omega t) \\
& -\frac{\epsilon_{1} A(\Phi) \omega+\left[\epsilon_{2} B(\Phi)+\epsilon_{3}\right] \gamma}{\omega\left(\gamma^{2}+\omega^{2}\right)} \cos (\omega t) .
\end{aligned}
$$

Notice that, if $A$ and $B$ are of order one, then $\zeta(t) \ll 1$ implies that $\left|\epsilon_{j}\right| \ll \omega^{2}+\gamma^{2}, j=1,2,3$.

Finally, we insert (9) into the remaining terms of Eq. (6) and take the average, defined by $\langle f(t)\rangle=\frac{1}{T} \int_{0}^{T} f(t) d t, T=$ $2 \pi / \omega$, of the resulting equation. Notice that since $\langle\zeta\rangle=0$, then $\langle\phi\rangle=\langle\Phi\rangle$. Using this fact, as well as the expressions

$$
\begin{aligned}
\left\langle\zeta^{2}\right\rangle & =\frac{\epsilon_{1}^{2} A^{2}(\Phi)+\left[\epsilon_{2} B(\Phi)+\epsilon_{3}\right]^{2}}{2 \omega^{2}\left(\omega^{2}+\gamma^{2}\right)}, \\
\langle\zeta \cos (\omega t)\rangle & =-\frac{\epsilon_{1} A(\Phi) \omega+\left[\epsilon_{2} B(\Phi)+\epsilon_{3}\right] \gamma}{2 \omega\left(\omega^{2}+\gamma^{2}\right)}, \\
\langle\zeta \sin (\omega t)\rangle & =\frac{\epsilon_{1} A(\Phi) \gamma-\left[\epsilon_{2} B(\Phi)+\epsilon_{3}\right] \omega}{2 \omega\left(\omega^{2}+\gamma^{2}\right)},
\end{aligned}
$$


the following averaging equation for $\langle\Phi\rangle$ is obtained:

$$
\begin{aligned}
\frac{\partial^{2} \Phi}{\partial t^{2}} & -\frac{\partial^{2} \Phi}{\partial x^{2}}+\gamma \frac{\partial \Phi}{\partial t}+V^{\prime}(\Phi) \\
& +\frac{\epsilon_{1}^{2} A^{2}(\Phi)+\left[\epsilon_{2} B(\Phi)+\epsilon_{3}\right]^{2}}{4 \omega^{2}\left(\omega^{2}+\gamma^{2}\right)} V^{\prime \prime \prime}(\Phi) \\
= & f-\epsilon_{1} A^{\prime}(\Phi) \frac{\epsilon_{1} A(\Phi) \omega+\left[\epsilon_{2} B(\Phi)+\epsilon_{3}\right] \gamma}{2 \omega\left(\omega^{2}+\gamma^{2}\right)} \\
& +\epsilon_{2} B^{\prime}(\Phi) \frac{\epsilon_{1} A(\Phi) \gamma-\left[\epsilon_{2} B(\Phi)+\epsilon_{3}\right] \omega}{2 \omega\left(\omega^{2}+\gamma^{2}\right)}
\end{aligned}
$$

Henceforth, for the sake of simplicity, the notation $\Phi=\langle\Phi\rangle$ is used. This is the averaging equation corresponding to Eq. (4). This has been obtained by the assumption that $\omega$ is sufficiently large, which means that the driving frequency should be larger than any other frequency of the system.

\section{DAMPED DOUBLE SINE-GORDON EQUATION, DRIVEN BY EXTERNAL (NONPARAMETRIC) AND PARAMETRIC FORCES OF HIGH FREQUENCY}

Let the results of the previous section now be applied to the damped double sine-Gordon equation driven by external (nonparametric) and parametric forces. To this end, the potential and the functions $A(\phi)$ and $B(\phi)$ in Eq. (4) are specified as follows:

$$
\begin{aligned}
V(\phi) & =\mu(1-\cos \phi)+\frac{\lambda}{2}(1-\cos 2 \phi), \\
A(\phi) & =\sin \phi, \quad B(\phi)=-\cos \phi,
\end{aligned}
$$

obtaining the equation

$$
\begin{aligned}
& \frac{\partial^{2} \phi}{\partial t^{2}}-\frac{\partial^{2} \phi}{\partial x^{2}}+\mu \sin \phi+\lambda \sin 2 \phi \\
& \quad=f-\gamma \frac{\partial \phi}{\partial t}+\epsilon_{1} \sin \phi \cos \omega t-\epsilon_{2} \cos (\phi) \sin \omega t+\epsilon_{3} \sin \omega t
\end{aligned}
$$

By maintaining only those terms up to the order $1 / \omega^{3}$, the effective Eq. (10) becomes

$$
\frac{\partial^{2} \Phi}{\partial t^{2}}-\frac{\partial^{2} \Phi}{\partial x^{2}}+\gamma \frac{\partial \Phi}{\partial t}+\frac{d V_{\mathrm{eff}}}{d \Phi}=f_{\mathrm{eff}},
$$

where

$$
\begin{aligned}
V_{\mathrm{eff}}(\Phi)= & {\left[\mu+\frac{\epsilon_{2} \epsilon_{3}}{2\left(\omega^{2}+\gamma^{2}\right)}\right](1-\cos \Phi) } \\
& +\left[\lambda+\frac{\epsilon_{1}^{2}-\epsilon_{2}^{2}}{4\left(\omega^{2}+\gamma^{2}\right)}\right]\left(\frac{1-\cos 2 \Phi}{2}\right) \\
& +\frac{\epsilon_{1} \epsilon_{3} \gamma}{2 \omega\left(\omega^{2}+\gamma^{2}\right)} \sin \Phi, \\
f_{\mathrm{eff}}= & f+\frac{\epsilon_{1} \epsilon_{2} \gamma}{2 \omega\left(\omega^{2}+\gamma^{2}\right)} .
\end{aligned}
$$

There are two interesting particular cases that deserve to be discussed:

(1) Parametrically driven damped sine-Gordon equation. Setting $\epsilon_{3}=0$ in Eq. (11) yields

$$
\begin{aligned}
& \frac{\partial^{2} \phi}{\partial t^{2}}-\frac{\partial^{2} \phi}{\partial x^{2}}+\mu \sin \phi+\lambda \sin 2 \phi \\
& \quad=f-\gamma \frac{\partial \phi}{\partial t}+\epsilon_{1} \sin \phi \cos \omega t-\epsilon_{2} \cos (\phi) \sin \omega t
\end{aligned}
$$

while the corresponding effective equation reduces to (12) with the effective potential and force given by

$$
\begin{aligned}
V_{\mathrm{eff}}(\Phi) & =\mu(1-\cos \Phi)+\left[\lambda+\frac{\epsilon_{1}^{2}-\epsilon_{2}^{2}}{4\left(\omega^{2}+\gamma^{2}\right)}\right] \frac{(1-\cos 2 \Phi)}{2} \\
f_{\mathrm{eff}} & =f+\frac{\epsilon_{1} \epsilon_{2} \gamma}{2 \omega\left(\omega^{2}+\gamma^{2}\right)},
\end{aligned}
$$

respectively. In particular, by setting $\mu=1, \epsilon_{2}=0$, and $\lambda=0$ in Eq. (15), the results of Ref. [20] for the parametrically driven damped sine-Gordon equation are recovered; that is, the effective equation is the double sine-Gordon system, where the existence of two connected $\pi$ kinks for a certain range of parameters is observed.

In order to show the importance of the second parametric force, notice that $\epsilon_{1}=\epsilon_{2}=\epsilon$ further reduces Eq. (14) to

$\frac{\partial^{2} \phi}{\partial t^{2}}-\frac{\partial^{2} \phi}{\partial x^{2}}+\mu \sin \phi+\lambda \sin 2 \phi=f-\gamma \frac{\partial \phi}{\partial t}+\epsilon \sin (\phi-\omega t)$,

while its effective equation reads

$$
\begin{aligned}
& \frac{\partial^{2} \Phi}{\partial t^{2}}-\frac{\partial^{2} \Phi}{\partial x^{2}}+\gamma \frac{\partial \Phi}{\partial t}+\mu \sin \Phi+\lambda \sin 2 \Phi \\
& =f+\frac{\epsilon^{2} \gamma}{2 \omega\left(\omega^{2}+\gamma^{2}\right)} .
\end{aligned}
$$

Equation (17) represents either the damped double sineGordon equation $(\mu \neq 0, \lambda \neq 0)$ or the damped sine-Gordon equation $(\mu \neq 0, \lambda=0)$, driven only by a constant effective force. It is well known that the solitary waves in these cases, after a transient time, reach a constant velocity and are stable [27]. This means that the second parametric driving can be used in a proper way in order to stabilize the kink in the parametrically driven $\phi^{4}$ equation [16]. Moreover, due to the parametric forces and damping, the motion caused by the external constant force can be reversed. Here, we consider small values of effective force so that kink-antikink pairs are not created.

(2) Damped sine-Gordon equation driven with additive periodic force [20]. This case is captured from our theory by setting $\mu=1, \epsilon_{1}=\epsilon_{2}=0$, and $\lambda=0$ in Eq. (11). Therefore, the effective equation reads

$$
\frac{\partial^{2} \Phi}{\partial t^{2}}-\frac{\partial^{2} \Phi}{\partial x^{2}}+\gamma \frac{\partial \Phi}{\partial t}+\left(1-\frac{\epsilon_{3}^{2}}{4 \omega^{4}}\right) \sin \Phi=f .
$$

Remarkably, the results obtained in Ref. [20] by using a different technique are recovered. Indeed, the effective equation obtained in Ref. [20] reads $\frac{\partial^{2} \Phi}{\partial t^{2}}-\frac{\partial^{2} \Phi}{\partial x^{2}}+\gamma \frac{\partial \Phi}{\partial t}+J_{0}\left(\frac{\epsilon_{3}}{\omega^{2}}\right)=f$, where $J_{0}$ denotes the Bessel function of the first kind. Using the expansion $J_{0}(x)=1-\frac{x^{2}}{4}+\cdots$, Eq. (18) is obtained. 

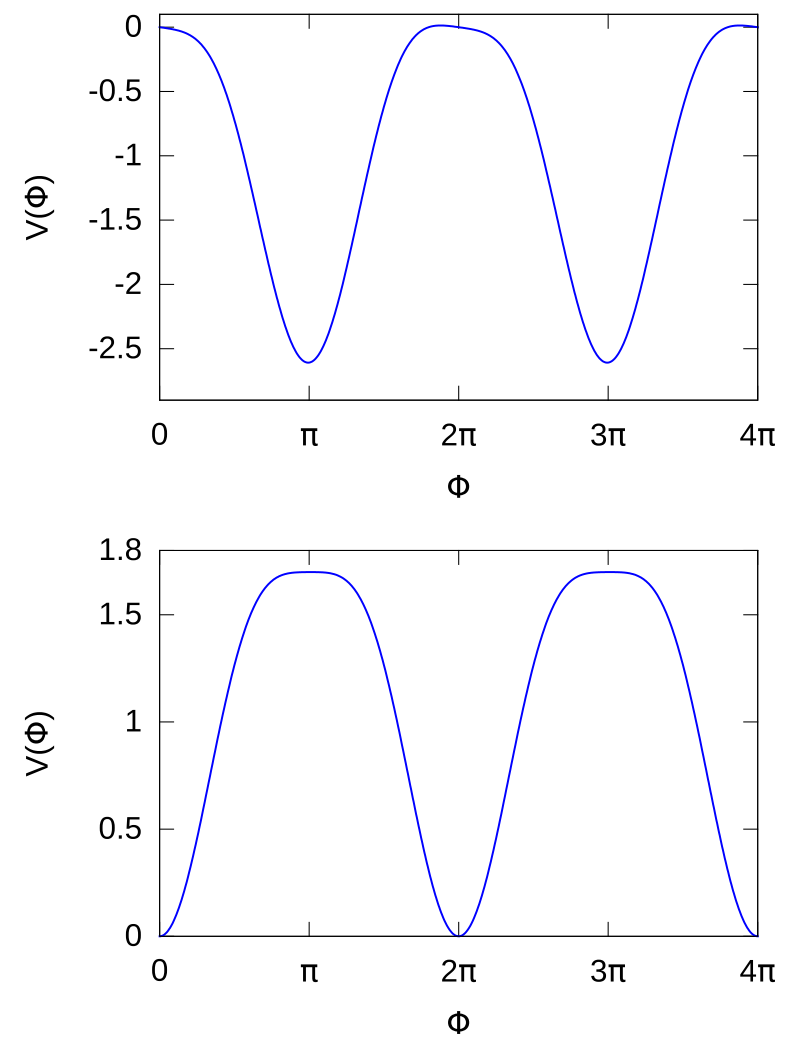

FIG. 1. (Color online) The effective potential (19) with $\epsilon_{3}=$ -50. Upper panel: $\omega=46$. Lower panel: $\omega=180$. Other parameters: $\mu=1, \lambda=2 / 5, \gamma=1, \epsilon_{1}=200$, and $\epsilon_{2}=195$.

Coming back to our general discussion, the different topologies are first analyzed of the kink solutions of Eq. (11) predicted from the analysis of Eqs. (12) and (13). The kink is a topological solitary wave that connects two minima of the nonlinear potential. Therefore, in order to study the different topologies of the kink induced by periodic forces of high frequency, we investigate how the minima of the effective potential can be controlled via frequency.

To facilitate this analysis, the effective potential (13) is rewritten as follows:

$$
\begin{aligned}
V_{\mathrm{eff}}(\Phi) & =a(\omega)(1-\cos \Phi)+b(\omega)\left(\frac{1-\cos 2 \Phi}{2}\right)+c(\omega) \sin \Phi \\
a(\omega) & =\mu+\frac{\epsilon_{2} \epsilon_{3}}{2\left(\omega^{2}+\gamma^{2}\right)} \\
b(\omega) & =\lambda+\frac{\epsilon_{1}^{2}-\epsilon_{2}^{2}}{4\left(\omega^{2}+\gamma^{2}\right)} \\
c(\omega) & =\frac{\epsilon_{1} \epsilon_{3} \gamma}{2 \omega\left(\omega^{2}+\gamma^{2}\right)} .
\end{aligned}
$$

Moreover, it is assumed that $\epsilon_{1}>\epsilon_{2}>0, \mu>0$, and $0<$ $\lambda<\mu / 2$. The study for other choices of the parameters can be carried out in a similar fashion.

Let us start by investigating the situation where no additive external force drives the system, i.e., $\epsilon_{3}=0$, implying $a=$ $\mu>0, b>0$, and $c=0$. If $\omega$ is sufficiently large, the original double sine-Gordon potential $\mu(1-\cos \Phi)+\frac{\lambda}{2}(1-\cos 2 \Phi)$ with $\lambda<\mu / 2$ is recovered. Thus the potential has the minima
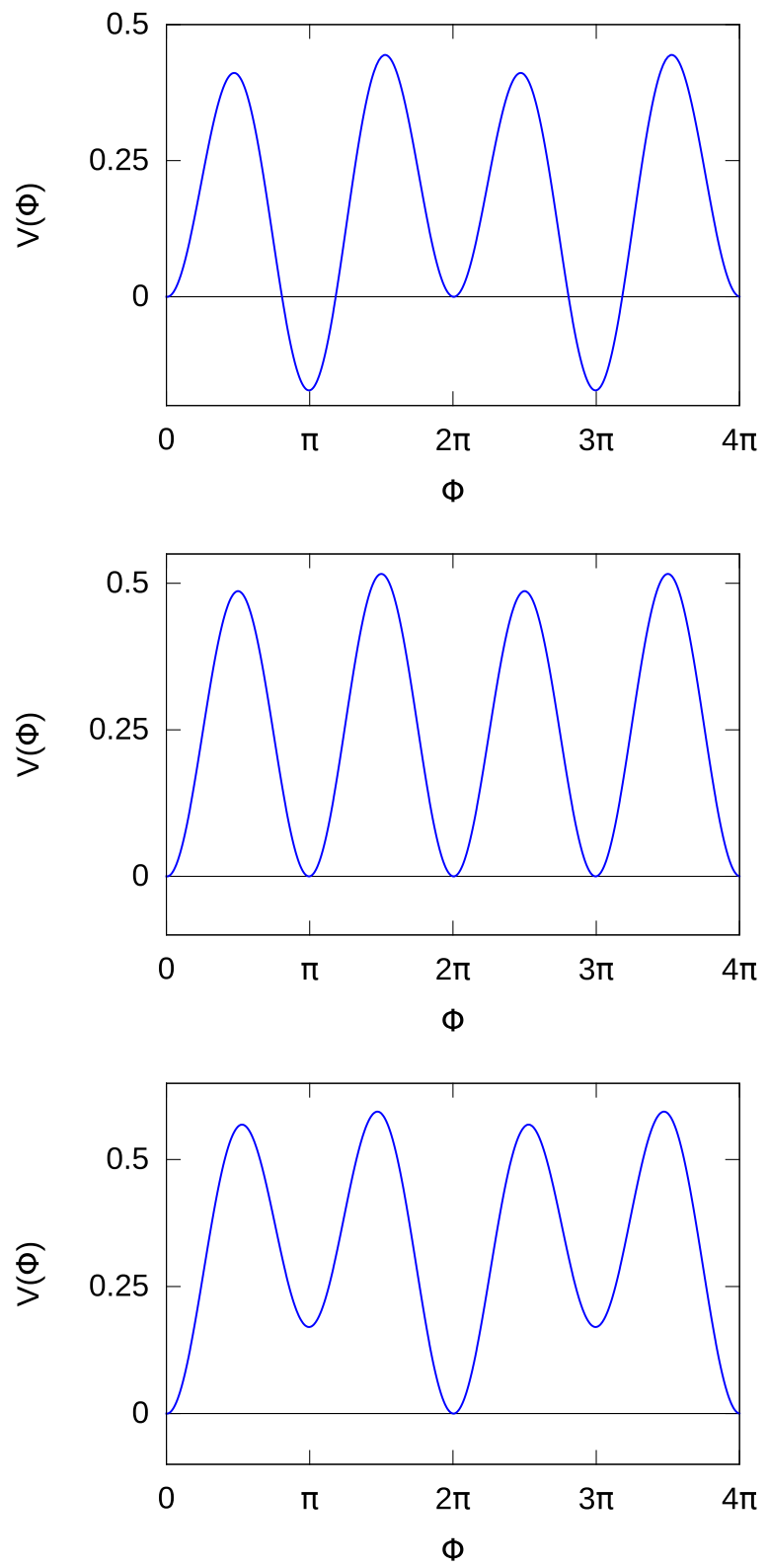

FIG. 2. (Color online) The effective potential (19) with $\epsilon_{3}=$ -50. Upper panel: $\omega=67$. Middle panel: $\omega_{c}=69.81$ (critical frequency to observe the two $\pi$ kinks). Lower panel: $\omega=73$. Other parameters: $\mu=1, \lambda=2 / 5, \gamma=1, \epsilon_{1}=200$, and $\epsilon_{2}=195$.

at 0 and $2 \pi$ and a kink connecting these values is expected. By decreasing the frequency $\omega$, a region can be reached, in which

$$
\lambda+\frac{\epsilon_{1}^{2}-\epsilon_{2}^{2}}{4\left(\omega^{2}+\gamma^{2}\right)}>\frac{\mu}{2} .
$$

In this case, the potential has absolute minima at 0 and $2 \pi$ and a relative minimum at $\pi$; a kink-kink solution connecting these three minima is therefore expected.

For the more general case, that of $\epsilon_{3} \neq 0$, the potential (19) always vanishes at $\Phi=0,2 \pi$. The minima of the potential needs to be computed. There are two possible choices of $\epsilon_{3}$ :

(1) $\epsilon_{3}>0$ : this implies $a(\omega)>0, b(\omega)>0$, and $c(\omega)>0$ in Eq. (19). Moreover, since $\omega$ is large, $c(\omega)$ will be a small quantity. This, together with the fact that $\mu$ and $\lambda$ are positive 

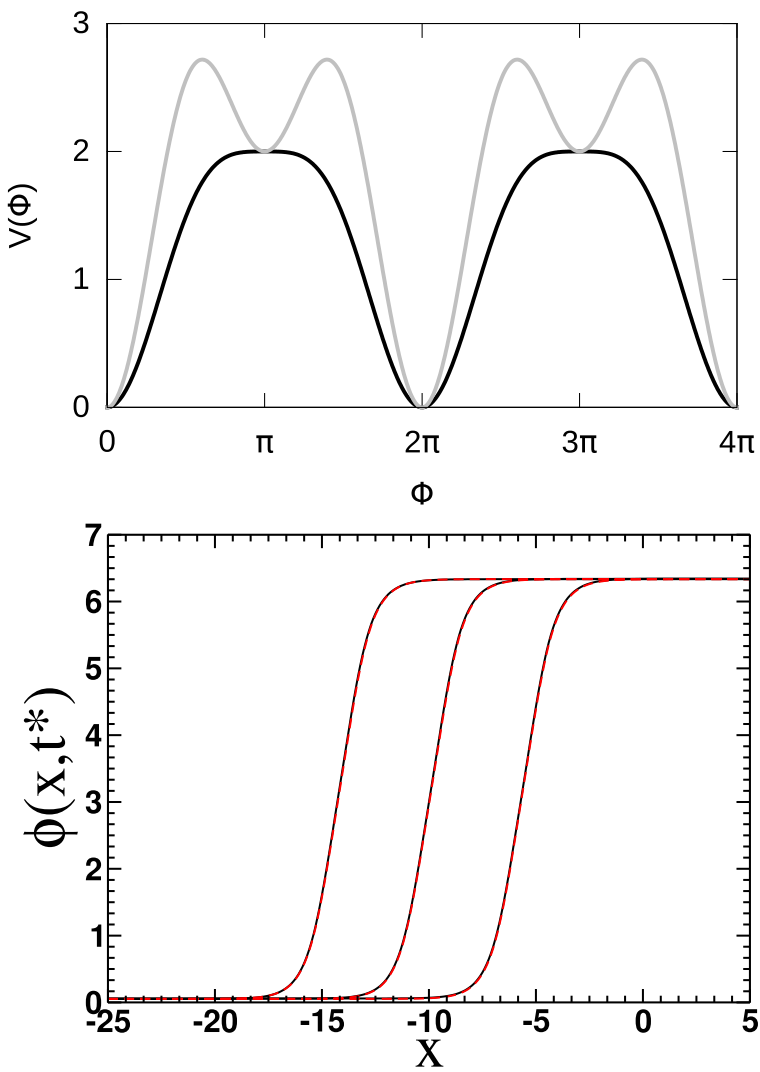

FIG. 3. (Color online) Upper panel: effective potential for $\omega=$ 160 (black solid line) and $\omega=45$ (gray solid line). Lower panel ( $\omega=$ 160): kink profiles moving to the left at $t^{*}=80,140,200$ obtained from simulations of the double sine-Gordon Eq. (11) (black solid line) and from the simulations of the effective Eqs. (12) and (13) (red dashed line). Other parameters: $\mu=1, \lambda=2 / 5, \gamma=1, f=0.1$, $\epsilon_{1}=200, \epsilon_{2}=175$, and $\epsilon_{3}=0$.

fixed numbers, guarantees that the potential is always positive (at least for sufficiently large $\omega$ ) and that it reaches its minima when $\Phi=0$ and $2 \pi$. Thus, for this specific choice, only one kink connecting 0 and $2 \pi$ exists.

(2) $\epsilon_{3}<0$ : this case is richer in comparison with the previous case because $a(\omega)$ can change its sign. Indeed, we have found the following three representative cases:

(a) The potential is negative and has maxima at 0 and $2 \pi$ when a frequency is chosen such that the function $a(\omega)<0$ and when the other parameters are fixed in such a way that the first term of the potential $a(\omega)[1-\cos (\Phi)]$ becomes the dominant one, i.e., $|a(\omega)| \gg|b(\omega)|$ (on choosing $\epsilon_{1} \approx \epsilon_{2}$ it is clear that this condition holds). Moreover, since $c(\omega)$ is very small ( $\omega$ is large), then a minimum is located close to $\pi$. A kink connecting the minima $\pi$ and $3 \pi$ is therefore expected. This case is represented in the upper panel of Fig. 1 for a given set of parameters.

(b) If we increase the frequency, $a(\omega)$ will increase and for a frequency $\omega \approx \omega_{c}=\sqrt{\epsilon_{2}\left|\epsilon_{3}\right| /(2 \mu)}$, then $a(\omega) \sim 0$. The second term is dominant, and the potential has an extra minimum at $\Phi \approx \pi$. Therefore, a kink-kink connecting $0, \pi$ and $2 \pi$ is expected if $V_{\text {eff }}(\pi)>0$, or a kink-kink connecting $\pi, 2 \pi$, and $3 \pi$ is expected if $V_{\text {eff }}(\pi)<0$. For $\omega=\omega_{c}$, the effective potential has global minima at $n \pi$; therefore the
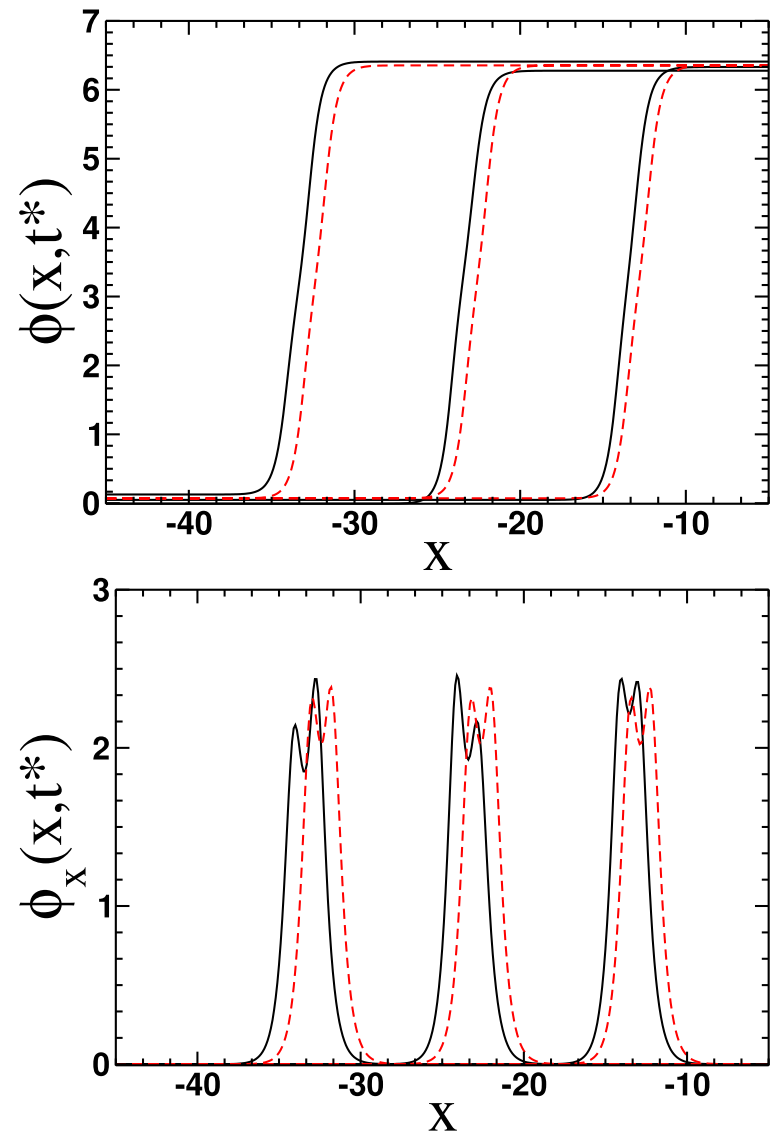

FIG. 4. (Color online) Upper and lower panels show kink profiles at $t^{*}=80,140,200$ and its spatial derivative at $t^{*}=80,200$, respectively, moving to the left obtained from simulations of Eq. (11) (black solid line) and from the simulations of the effective Eqs. (12) and (13) (red dashed line). Other parameters: $\lambda=2 / 5, \gamma=1, f=0.1$, $\epsilon_{1}=200, \epsilon_{2}=175, \epsilon_{3}=0$, and $\omega=45$.

appearance of two slightly different $\pi$ kinks is expected for this specific value of the frequency: one $\pi$ kink connects 0 and $\pi$, and the other connects $\pi$ and $2 \pi$ (see Fig. 2).

(c) Finally, for a very large frequency, one recovers the double sine-Gordon potential $\mu(1-\cos \Phi)+\frac{\lambda}{2}(1-$ $\cos 2 \Phi)$, which with our choice of parameters has two global minima at 0 and $2 \pi$. Therefore, since $\lambda<\mu / 2$, from the results in Ref. [3] one expects a kink connecting 0 and $2 \pi$ (see lower panel of Fig. 1).

\section{NUMERICAL SIMULATIONS}

In order to check the results of the previous section, simulations of Eq. (11) and the effective Eqs. (12) and (13) are performed by using a fourth-order Runge-Kutta method with spatial discretization step $\Delta x=0.1$, time discretization step $\Delta t=0.01 ; 0.001$, and length of the system $L=100$. To solve Eq. (11) and Eqs. (12) and (13), we mostly use the static kink solution $\phi_{0}$ given in the Appendix A as the initial condition with the appropriate parameters $\mu$ and $\lambda$. In the case when the periodic effective potential presents two global minima between two global maxima (see upper panel in Fig. 2 and also Fig. 6), we start from the double sine-Gordon 

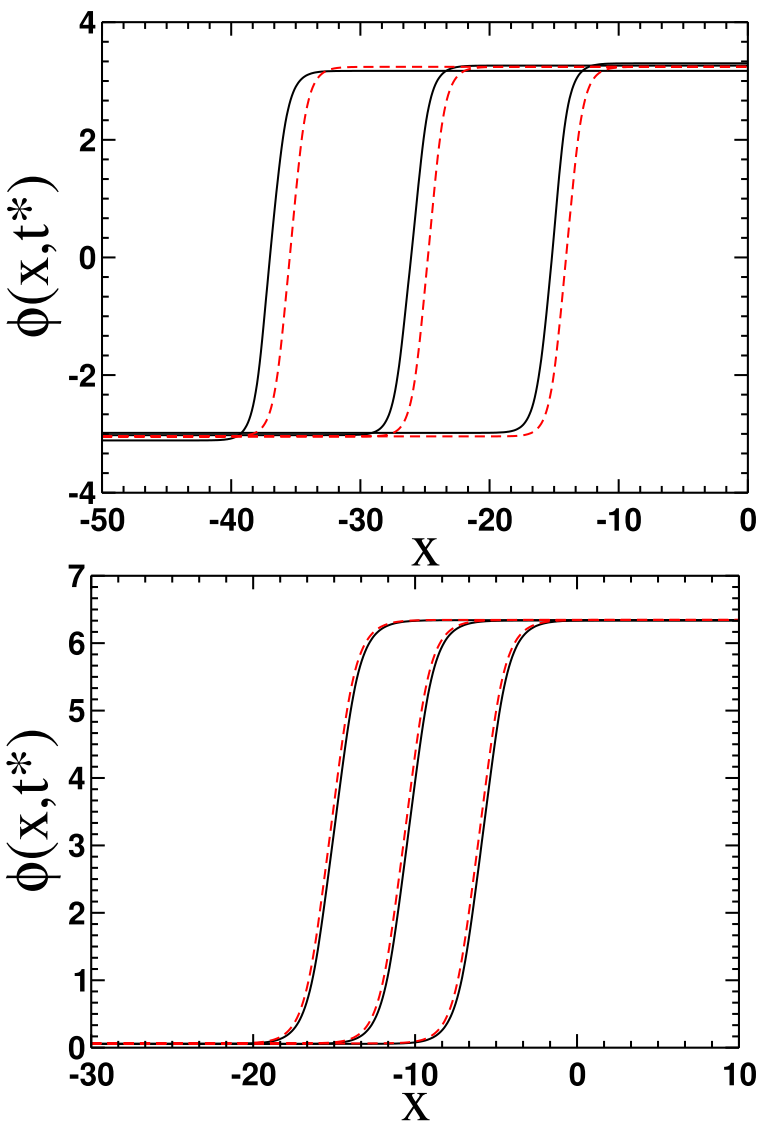

FIG. 5. (Color online) Upper and lower panels show kink profiles, moving to the left, at $t^{*}=80,140,200$ for $\omega=46$ and $\omega=$ 180, respectively. Black solid lines: simulations of Eq. (11). Red dashed lines: simulations of the effective Eqs. (12) and (13). Other parameters: $\mu=1, \lambda=2 / 5, \gamma=1, f=0.1, \epsilon_{1}=200, \epsilon_{2}=195$, and $\epsilon_{3}=-50$.

static kink solution which connects 0 and $2 \pi$ (two consecutive relative minima of the effective potential). However, the initial condition evolves quickly to a kink connecting $-\pi$ and $\pi$ (two consecutive global minima of the effective potential). This fact has been used in the simulations of Eq. (11) in Fig. 8, where the initial static kink solution has been shifted by $\pi$. Moreover, we fix $\mu=1, \lambda=2 / 5, \gamma=1$, and $f=0.1$, and choose $\omega \in[44,180]$ and $\left|\epsilon_{j}\right| \in[50,200]$ in all simulations of this section.

\section{A. Case $\epsilon_{1} \neq \epsilon_{2}$ and $\epsilon_{3}=0$}

Setting $\epsilon_{3}=0$, in the previous section we showed that, by decreasing the high frequency, the two $\pi$ kink solutions should be observed (see upper panel in Fig. 3). These predictions are confirmed by simulations presented in Figs. 3 and 4. For $\omega=160$ we observe, in the lower panel of Fig. 3 , a $2 \pi$ kink traveling to the left. By decreasing the frequency, the two $\pi$ kink solutions in Fig. 4 can be identified, where the first spatial derivative of the kink profile is shown (lower panel in Fig. 4). The distance between the centers of the two $\pi$ kinks is very small (around 1), and for this reason they are hardly visible in the kink profiles (upper panel in Fig. 4). However, the two peaks of the function $\phi_{x}$, represented in the lower panel of Fig. 4, clearly provide evidence of the existence of the two

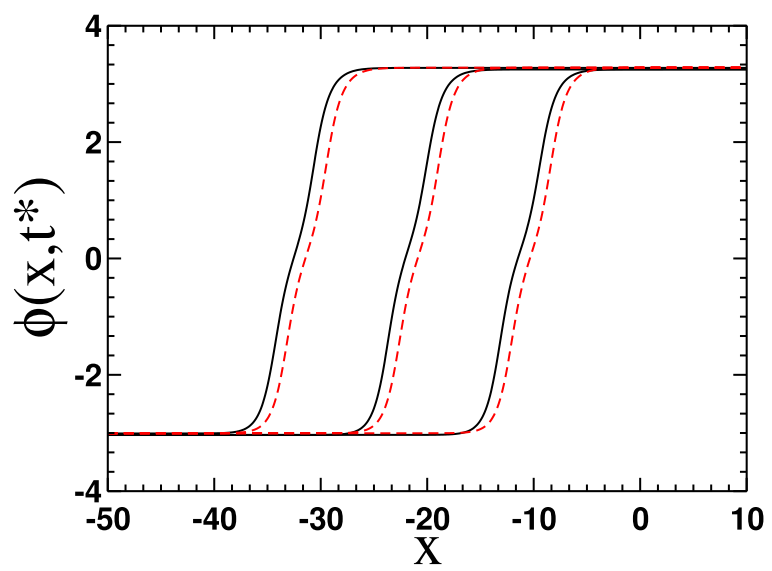

FIG. 6. (Color online) Kink profiles moving to the left at $t^{*}=$ 50,100,150 for $\omega=67 \lesssim \omega_{c}=69.81$. Black solid lines: simulations of Eq. (11). Red dashed lines: simulations of the effective Eqs. (12) and (13). Parameters of the simulations: $\lambda=2 / 5, \gamma=1, f=0.1$, $\epsilon_{1}=200, \epsilon_{2}=195, \epsilon_{3}=-50$.

$\pi$ kinks. The shift that is observed (between dashed and solid lines in Fig. 4) is due to the dynamics of Eqs. (11) and (12) and (13) during the transient time. Indeed, after a transient time, it is verified that the kink solutions of both equations travel with practically the same velocity, $v=-0.163 \approx-0.165$. By means of increasing the frequency from $\omega=45$ to $\omega=180$, and fixing all the parameters as in Figs. 3 and 4, we find that this shift between the two kinks tends to disappear and vanishes when the frequencies are greater than 100 .

\section{B. Case $\epsilon_{1} \neq \epsilon_{2}$ and $\epsilon_{3} \neq 0$}

The results for $\epsilon_{3}<0$ are presented in Figs. 5-8 for three different frequencies, which represent the three different cases $\omega \in[44,180]$, as discussed in the previous section. In all these figures we observe a very good agreement between the simulations of the original partial differential equation and its corresponding effective equation.

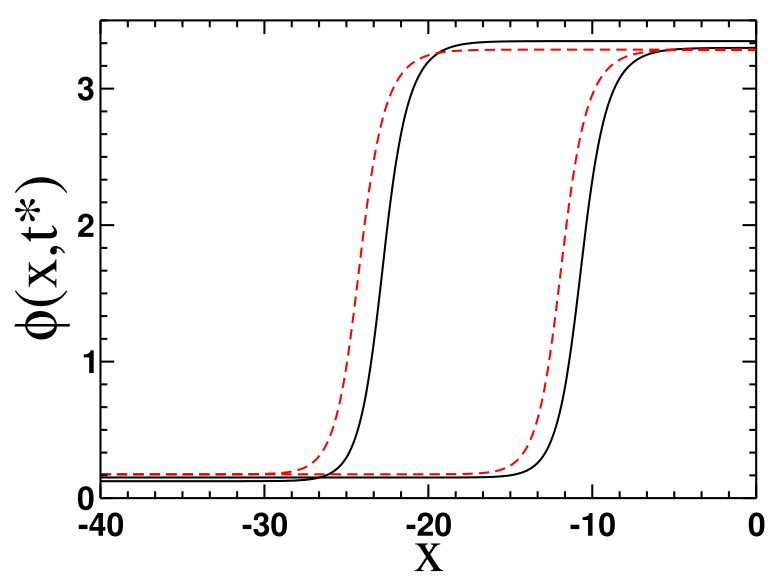

FIG. 7. (Color online) Kink profiles moving to the left at $t^{*}=$ 50,100 for $\omega=\omega_{c}=69.81$. Black solid lines: simulations of Eq. (11). Red dashed lines: simulations of the effective Eqs. (12) and (13). Parameters of the simulations: $\lambda=2 / 5, \gamma=1, f=0.1$, $\epsilon_{1}=200, \epsilon_{2}=195, \epsilon_{3}=-50$. 


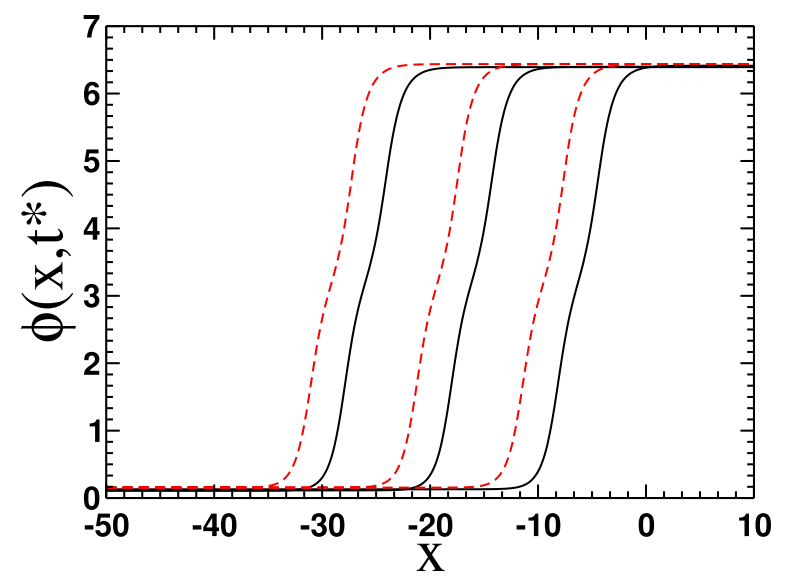

FIG. 8. (Color online) Kink profiles moving to the left at $t^{*}=$ 50,100,150 for $\omega=73 \gtrsim \omega_{c}=69.81$. Black solid lines: simulations of the Eq. (11). Red dashed lines: simulations of the effective Eqs. (12) and (13). Parameters of the simulations: $\lambda=2 / 5, \gamma=1, f=0.1$, $\epsilon_{1}=200, \epsilon_{2}=195, \epsilon_{3}=-50$.

Relatively low and high frequencies are chosen in Fig. 5, where for each frequency the $2 \pi$ kinks connect different minima of the potential. As expected from the averaging method, it is observed that the mobility of the kink is reduced by increasing the frequency.

By fixing all the parameters as in Fig. 5 and changing the frequency, we approach the critical frequency $\omega_{c}=69.81$. In this case, a $\pi$ kink is observed (Fig. 7), because the minima of the effective potential are at $n \pi$. In order to obtain this kink, we start from the static kink solution of the sine-Gordon equation, $\phi_{0}(x)=2 \arctan [\exp (x)]$ in the simulations of the effective equation. In simulations of the double sine-Gordon model, this initial condition has been shifted by $\pi / 2$. Below and above this critical frequency, the two $\pi$ kinks are predicted and confirmed by the simulations in Figs. 6 and 8 .

\section{ABSOLUTE NEGATIVE MOBILITY}

An intriguing phenomenon, predicted by the averaging method, is related to the possibility of the inversion of direction of the motion by changing the frequency or the amplitudes of the periodic drivers (this effect has been observed in the context of soliton ratchets [28,29]). Indeed, Eqs. (12) and (13) show that the kink is subject to an effective constant force, given by

$$
f_{\text {eff }}=f+\frac{\epsilon_{1} \epsilon_{2} \gamma}{2 \omega\left(\omega^{2}+\gamma^{2}\right)} .
$$

For positive (negative) values of the effective force in Eq. (21), the kink moves to the left (right). Since our analysis has fixed $\epsilon_{1}>0$ and $\epsilon_{2}>0$, the effective force could be zero only if $f<0$. This means that, by setting $f<0$, the frequency, the damping and amplitudes can be changed such that the effective force is positive and the kink moves to the left (see Fig. 9). Indeed, by fixing all the parameters as in Fig. 9 and by changing $\omega \in[50,60]$, it is obtained from Eq. (21) that the motion is suppressed when $\omega=57.98$. This result is well confirmed by the comparison between simulations of Eq. (11) and simulations of the effective Eqs. (12) and (13); see Fig. 10. Here it worth pointing out the role of the damping as well as
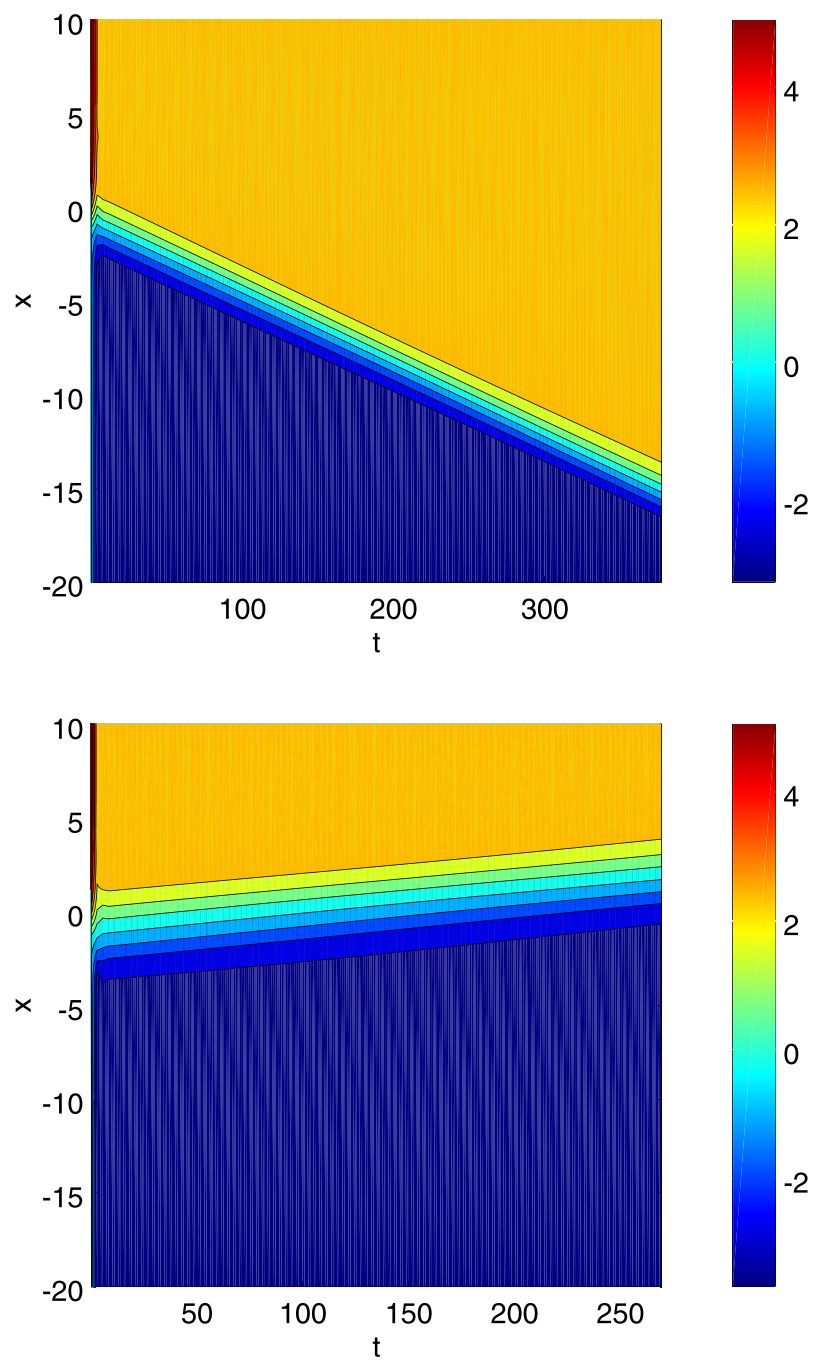

FIG. 9. (Color online) Upper and lower panels: contour plot of the kink obtained from simulations of Eq. (11), for $\omega=50$ and $\omega=$ 60 , respectively. Parameters of the simulations: $\lambda=2 / 5, \gamma=1, f=$ $-0.1, \epsilon_{1}=200, \epsilon_{2}=195, \epsilon_{3}=-50$.

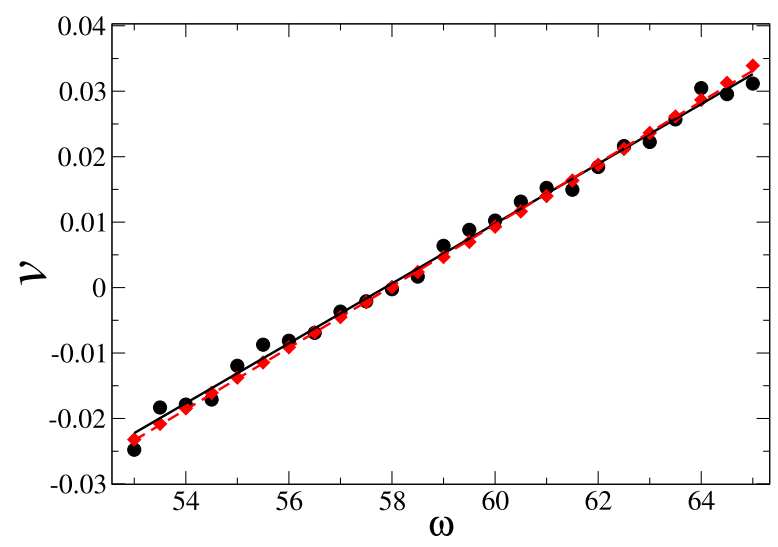

FIG. 10. (Color online) Upper and lower panels: kink velocity versus frequency, $\omega$. Black circles and red diamonds: results from simulations of Eq. (11) and Eqs. (12) and (13), respectively. These results are fitted with linear functions represented by a black solid line and a red dashed line, respectively. Parameters of the simulations: $\lambda=2 / 5, \gamma=1, f=-0.1, \epsilon_{1}=200, \epsilon_{2}=195, \epsilon_{3}=-50$. 
that of the two parametric forces on the effective driving force $f_{\text {eff }}$. When damping approaches to zero or when one of the two parametric forces is not present, then the direction of the motion cannot be reversed once $f \rightarrow f_{\text {eff }}$.

\section{SUMMARY}

We have studied the damped double sine-Gordon equation driven by a constant, an external (nonparametric) $T=2 \pi / \omega$ periodic force, and two parametric $T$ periodic forces, for sufficiently large values of $\omega$. The dynamics of the kink is investigated by means of the so-called averaging method [16], which was first developed for the parametrically driven $\phi^{4}$ system.

The method describes an approximate solution of the system by means of two functions: one function is a homogeneous fast-varying function, and the other is a slowly varying function. The dynamics of the perturbed double sine-Gordon is then mapped to the dynamics of an effective double sine-Gordon equation, with nonperiodic forces, but with an effective potential and an effective constant force. By varying the amplitudes, the damping and the frequencies of the periodic forces, not only is the effective potential changed, but also the effective force. In particular, these changes affect the topology of the kink and its mobility.

For high-frequency, the simulations of the original perturbed double sine-Gordon equation agree with the simulations of the effective equation. We have also used the simulations in order to verify the assumptions of the averaging method.

Particular attention has been devoted to the investigation of the topology of the kinks, namely, the formation of the $\pi$ kink and the appearance of two $\pi$ kinks. These phenomena have been reported before for two different systems [20,23]; these two types of kinks can now emerge in one system and can be controlled via frequency.

Remarkably, we have found that the direction of the motion of the kink is not only dictated by the external constant force of the double sine-Gordon equation, but that it can also be changed by varying the frequency and amplitudes of external (nonparametric) and parametric periodic forces, and by varying the damping. This phenomenon is similar to the anomalous negative mobility (current flows in the opposite direction to the applied constant force) that has been observed experimentally in semiconductor superlattices [30] and in Josephson junctions [31].

\section{ACKNOWLEDGMENTS}

We thank the referees for their useful comments and suggestions on this work. Financial support was received through grants MTM2012-36732-C03-03 (R.A.N.) and FIS201124540 (N.R.Q.) from the Ministerio de Economía y Competitividad (Spain), grants FQM262 (R.A.N.), FQM207 (N.R.Q.), FQM-7276, and P09-FQM-4643 (N.R.Q., R.A.N.) from the Junta de Andalucía (Spain), and a grant from the Humboldt Foundation through the Research Fellowship for Experienced Researchers SPA 1146358 STP (N.R.Q.).

\section{APPENDIX: LINEAR SPECTRUM AROUND THE STATIC KINK SOLUTION IN THE DOUBLE SINE-GORDON EQUATION}

It is well known that, for $|\lambda|<|\mu| / 2$, the static kink solution of the double sine-Gordon equation [i.e., Eq. (1) with $f=0$, $\gamma=0$ and $\left.\epsilon_{1}=\epsilon_{2}=\epsilon_{3}=0\right]$ is represented by

$$
\phi_{0}(x)= \begin{cases}-2 \arctan \sqrt{2 \lambda / \mu+1}(\sinh \sqrt{2 \lambda+\mu} x)^{-1}, & x \leqslant 0 \\ 2 \pi-2 \arctan \sqrt{2 \lambda / \mu+1}(\sinh \sqrt{2 \lambda+\mu} x)^{-1}, & x>0\end{cases}
$$

The stability of the kink solution in the double sine-Gordon equation was numerically studied in Refs. [13,32] (see also the references therein). We follow a standard procedure developed in Ref. [33] for the sine-Gordon equation. Since the unperturbed double sine-Gordon equation is Lorentz invariant, without loss of generality, it can be linearized around its exact static kink solution, $\phi_{0}(x)$ [Eq. (A1)]; i.e., it is assumed that the solution of the unperturbed double sine-Gordon is $\phi_{0}(x)+\psi(x, t)$, where $|\psi(x, t)| \ll 1$ [33]. This leads us to the following linear problem for $\psi(x, t)$ :

$$
\begin{array}{r}
\psi_{t t}-\psi_{x x}+\left[\mu \cos \left(\phi_{0}\right)+2 \lambda \cos \left(2 \phi_{0}\right)\right] \psi=0, \\
-\infty<x<\infty, \quad \psi_{x}( \pm \infty)=0,
\end{array}
$$

where $L$ is the length of the system, whose solution can be expressed as

$$
\psi(x, t)=\exp (i \Omega t) h(x), \quad h_{x}( \pm \infty)=0 .
$$

Second, by substituting Eq. (A3) into Eq. (A2), it can finally be concluded that $h(x)$ satisfies

$$
\begin{array}{r}
h_{x x}+\left[\Omega^{2}-\mu \cos \left(\phi_{1}\right)-2 \lambda \cos \left(2 \phi_{1}\right)\right] h=0, \\
-\infty<x<+\infty, \quad h_{x}( \pm \infty)=0 .
\end{array}
$$

For the sine-Gordon equation, i.e., when $\lambda=0$, this eigenvalue problem can be exactly solved [33]. When $\lambda \neq 0$, this equation can be numerically solved for any finite length of the system.

By using the QR algorithm, we have numerically solved Eq. (A4), by fixing $L=100, \Delta x=0.1$, and by taking $\mu=1$ and $\lambda=2 / 5$. The spectrum contains the Goldstone mode close to $\Omega=0$, the internal mode frequency below the phonon band $\Omega_{\text {min }}=\sqrt{\mu+2 \lambda}=1.34$, and the frequencies of the phonons between $\Omega_{\min }$ and $\Omega_{\max }=20.045$.
[1] A. C. Scott, Nonlinear Science (Oxford University, Oxford, 1999).
[2] L. V. Yakushevich, Nonlinear Physics of DNA (John Wiley and Sons, New York, 1998). 
[3] C. A. Condat, R. A. Guyer, and M. D. Miller, Phys. Rev. B 27, 474 (1983).

[4] D. K. Campbell, J. F. Schonfeld, and C. A. Wingate, Physica D 9, 1 (1983).

[5] M. Peyrard and D. K. Campbell, Physica D 9, 33 (1983).

[6] D. K. Campbell and M. Peyrard, Physica D 18, 47 (1986).

[7] A. V. Savin, Y. Zolotaryuk, and J. C. Eilbeck, Physica D 138, 267 (2000).

[8] N. R. Quintero and P. G. Kevrekidis, Physica D 170, 31 (2002).

[9] A. Sánchez and A. R. Bishop, SIAM Rev. 40, 579 (1998).

[10] A. V. Ustinov, C. Coqui, A. Kemp, Y. Zolotaryuk, and M. Salerno, Phys. Rev. Lett. 93, 087001 (2004).

[11] M. Beck, E. Goldobin, M. Neuhaus, M. Siegel, R. Kleiner, and D. Koelle, Phys. Rev. Lett. 95, 090603 (2005).

[12] Yu. S. Kivshar and B. A. Malomed, Rev. Mod. Phys. 61, 763 (1989).

[13] D. K. Campbell, M. Peyrard, and P. Sodano, Physica D 19, 165 (1986).

[14] S. Pnevmatikos, Phys. Rev. Lett. 60, 1534 (1988).

[15] A. Zolotaryuk and S. Pnevmatikos, Phys. Lett. A 143, 233 (1990).

[16] Yu. S. Kivshar, A. Sánchez, and L. Vázquez, Phys. Rev. A 45, 1207 (1992).

[17] E. Zamora-Sillero, N. R. Quintero, and F. G. Mertens, Phys. Rev. E 74, 046607 (2006).

[18] Yu. S. Kivshar, N. Gronbech-Jensen, and M. R. Samuelsen, Phys. Rev. B 45, 7789 (1992).

[19] N. Gronbech-Jensen, Yu. S. Kivshar, and M. Salerno, Phys. Rev. Lett. 70, 3181 (1993).
[20] Yu. S. Kivshar, N. Gronbech-Jensen, and R. D. Parmentier, Phys. Rev. E 49, 4542 (1994).

[21] Yu. S. Kivshar and K. H. Spatschek, Chaos Solitons Fractals 5, 2551 (1995).

[22] Yu. S. Kivshar and A. Sánchez, Phys. Rev. Lett. 77, 582 (1996).

[23] V. Zharnitsky, I. Mitkov, and N. Gronbech-Jensen, Phys. Rev. E 58, R52 (1998).

[24] N. R. Quintero and A. Sánchez, Eur. Phys. J. B 6, 133 (1998).

[25] N. Casic, N. R. Quintero, R. Alvarez-Nodarse, F. G. Mertens, L. Jibuti, W. Zimmermann, and T. M. Fischer, Phys. Rev. Lett. 110, 168302 (2013).

[26] L. D. Landau and M. Lifshitz, Mechanics (Pergamon Press, Oxford, 1960).

[27] D. W. McLaughlin and A. C. Scott, Phys. Rev. A 18, 1652 (1978).

[28] M. Salerno and Y. Zolotaryuk, Phys. Rev. E 65, 056603 (2002).

[29] J. A. Cuesta, N. R. Quintero, and R. Álvarez-Nodarse, Phys. Rev. X 3, 041014 (2013).

[30] B. J. Keay, S. Zeuner, S. J. Allen, Jr., K. D. Maranowski, A. C. Gossard, U. Bhattacharya, and M. J. W. Rodwell, Phys. Rev. Lett. 75, 4102 (1995).

[31] J. Nagel, D. Speer, T. Gaber, A. Sterck, R. Eichhorn, P. Reimann, K. Ilin, M. Siegel, D. Koelle, and R. Kleiner, Phys. Rev. Lett. 100, 217001 (2008).

[32] J. A. Holyst and H. Benner, Phys. Rev. E 52, 4583 (1995).

[33] M. B. Fogel, S. E. Trullinger, A. R. Bishop, and J. A. Krumhansl, Phys. Rev. B 15, 1578 (1977). 